\title{
Evolution of Superconducting String Currents
}

\author{
C. J. A. P. Martins* and E. P. S. Shellard ${ }^{\dagger}$ \\ Department of Applied Mathematics and Theoretical Physics \\ University of Cambridge \\ Silver Street, Cambridge CB3 9EW, U.K.
}

(22 May 1997)

\begin{abstract}
We extend the quantititative string evolution model of Martins and Shellard to superconducting strings by introducing a simple toy model for the evolution of the currents. This is based on the dynamics of a 'superconducting correlation length'. We derive the relevant evolution equations and discuss the importance of plasma effects. Some consequences of our results are also suggested.
\end{abstract}

PACS: $98.80 . \mathrm{Cq}, 11.27 .+\mathrm{d}$

Keywords: cosmology; cosmic string evolution; superconducting currents

\section{INTRODUCTION}

It is well known that cosmic strings can in some circumstances (typically when the electromagnetic gauge invariance is broken inside the string) behave as 'superconducting wires' carrying large currents and charges - up to the order of the string mass scale in appropriate units [1]. The charge carriers can be either bosons or fermions (see [2] for a review). The former type occurs when it becomes energetically favourable for a charged Higgs field to have a non-zero vacuum expectation value in the string core; the latter happens when there are fermion zero modes moving in the string field.

If superconducting strings carry currents, they must also carry charges of similar magnitude. This includes not only charges trapped at formation by the Kibble mechanism but also the ones due to string inter-commuting between regions of the string network with different currents. However, arbitrarily large currents or charges are not allowed - there are critical values beyond which the current saturates and charge carriers can leave the string.

Even though the overwhelming majority of the work done on cosmic strings so far was concerned with the structureless Goto-Nambu strings, there are a number of cosmological scenarios in which superconducting strings play a crucial partnotably the so-called 'vorton problem' [3] - so that their study is highly relevant.

In this paper we take a first step in this direction by generalizing the quantitative string evolution model of Martins and Shellard [4]. After a brief review of this model, we extend it by developing a simple toy model for the evolution of the currents on the long strings, based on the dynamics of a 'superconducting correlation length'. The different contributing mechanisms are discussed, and in particular we consider the importance of plasma effects. Finally, we briefly mention some of the implications of our model - a more detailed discussion is left for a forthcoming publicatiom $[9]$.

\footnotetext{
*Also at C. A. U. P., Rua do Campo Alegre 823, 4150 Porto, Portugal. Electronic address: C.J.A.P.Martins @ damtp.cam.ac.uk

${ }^{\dagger}$ Electronic address: E.P.S.Shellard @ damtp.cam.ac.uk

Submitted to Phys. Lett. B.
} 
Throughout this paper we will use fundamental units in which $\hbar=c=k_{B}=$ $G m_{P l}^{2}=1$.

\section{EVOLUTION OF THE CURRENTS}

Analytic string evolution methods describe the string network by a small number of macroscopic quantities whose evolution equations are derived from the microscopic string equations of motion. The first such model providing a quantitative picture of the complete evolution of a string network (and the corresponding loop population) has been recently developed by Martins and Shellard [4. such quantities, the long-string correlation length $\rho_{\infty} \equiv \mu / L^{2}$ ( $\mu$ being the string mass per unit length) and the string RMS velocity, $v^{2} \equiv\left\langle\dot{\mathbf{x}}^{2}\right\rangle$. It also includes two 'phenomenological' terms, a 'loop chopping efficiency' parameter $0<\tilde{c}<1 / 2$ and a 'small-scale structure parameter' $0<k<1$. Their evolution equations are

$$
\begin{aligned}
& 2 \frac{d L}{d t}=2 H L\left(1+v_{\infty}^{2}\right)+v_{\infty}^{2} \frac{L}{\ell_{f}}+\tilde{c} v_{\infty}, \\
& \frac{d v}{d t}=\left(1-v^{2}\right)\left[\frac{k}{L}-v\left(2 H+\frac{1}{\ell_{f}}\right)\right] ;
\end{aligned}
$$

these are sufficient to quantitatively describe the large-scale properties of a cosmic string network.

Note that the explicit form of the 'friction lengthscale' $\ell_{\mathrm{f}}$ will depend on the type

of current involved. For the case of a neutral current, one expects Aharonov-Bohm scattering 10] to be the dominant effect, and consequently we have [5]

$$
\ell_{\mathrm{f}}=\frac{\mu}{\beta T^{3}},
$$

where $T$ is the background temperature and $\beta$ is a numerical factor related to the number of particle species interacting with the string. We assume that this is the case for the time being, but the more general case where plasma effects can be important will be discussed towards the end of this paper.

We now extend it setting up a 'toy model' for the evolution of the currents. One assumes that there is a 'superconducting correlation length', denoted $\xi$, which measures the scale over which one has coherent current and charge densities on the strings. Associated with this we can define $N$ to be the number of uncorrelated current regions (in the long-string network) in a co-moving volume $V$, and it is then fairly straightforward to see how the dynamics of the string network affects $N$ and obtain an evolution equation for it.

Firstly, we expect that in a co-moving volume the number of uncorrelated regions will not be affected by expansion. Now consider the effect of inter-commutings (whether or not a loop is produced). Their effect on $N$ can easily be obtained by multiplying the inter-commuting rate (obtained from the original analytical model [5]) by the number of regions created by each inter-commuting - this will obviously be a positive term. Laguna and Matzner [11] have numerically shown that whenever two current-carrying strings cross, they inter-commute and a region of intermediate current is created. This means that inter-commutings will in general create four new regions (see figure 1 (a)). An exception to this is that when regions with size of order $\xi$ or smaller self-intersect it is possible (see figure 1 (b-c)) that no new regions are produced. Also, when the inter-commuting does produce a loop, the regions in the corresponding segment are removed from the network, together with one of the 
newly created 'intermediate' regions, so that there will be a negative term in the evolution of $N$ (with a similar correction factor at small scales).

Finally, the only non-trivial issue is that of the dynamics of the currents themselves. The simulations of Laguna and Matzner [11] show that as the result of inter-commutings charges pile up at current discontinuities and move with the kinks, but their strength decreases with time. Clearly, this indicates that some kind of 'equilibration' process should act between neighbouring current regions, which will counteract the creation of new regions by inter-commuting (and help the removal of regions by loops). Furthermore, Austin, Copeland and Kibble have shown [12] that in an expanding universe correlations between left- and right-moving modes develop due both to stretching and inter-commuting (particularly when loops form). We model this term by assuming that after each Hubble time, a fraction $f$ of the $N$ regions existing at its start will have equilibrated with one of its neighbours,

$$
\left(\frac{d N}{d t}\right)_{\text {dynamics }}=-f H N
$$

note that new regions are obviously created by inter-commuting during the Hubble time in question, so that $f$ can be larger than unity. Alternatively we can say that for a given $f$, the number of regions that were present in a given volume at a time $t$ will have disappeared due to equilibration at a time $t+(f H)^{-1}$. Presumably the only way to find out what $f$ is is by means on numerical simulations (in particular, we would expect it to be a model-dependent quantity), although some physical arguments can be used to constrain it [9].

However, setting this issue aside for the time being, we obtain the following evolution equation for $N$

$$
\frac{d N}{d t}=G\left(\frac{\ell}{\xi}\right) \frac{v_{\infty}}{\alpha} \frac{V}{L^{4}}-f H N
$$

where the 'correction factor' $G$ has the form

$$
G\left(\frac{\ell}{\xi}\right)=\left\{\begin{array}{ll}
2-\tilde{c}\left(\frac{\ell}{\xi}+2\right), & \frac{\ell}{\xi}>1 \\
2(1-2 \tilde{c}) \alpha+(2-3 \tilde{c}-2 \alpha+4 \alpha \tilde{c}) \frac{\ell}{\xi}, & \frac{\ell}{\xi} \leq 1
\end{array} ;\right.
$$

loops are assumed to form with a size $\ell(t)=\alpha(t) L(t)$, with $\alpha \sim 1$ while the string network is is the friction-dominated epoch and $\alpha=\alpha_{s c} \ll 1$ once it has reached the linear scaling regime (see [5]). Note that when $\ell \gg \xi$ the net effect of intercommuting and loop production is to remove uncorrelated regions (because each loop formed removes a large number of them); otherwise, the net effect is to create new regions.

For what follows it is more convenient to introduce $N_{L}$, defined to be the number of uncorrelated current regions per long-string correlation length,

$$
N_{L} \equiv \frac{L}{\xi}
$$

in terms of $N_{L},(2.5)$ has the form

$$
\frac{d N_{L}}{d t}=\left(3 v_{\infty}^{2}-f\right) H N_{L}+\frac{3}{2} \frac{v_{\infty}^{2}}{\ell_{\mathrm{f}}} N_{L}+\left(\frac{1}{\alpha} G\left(\alpha N_{L}\right)+\frac{3}{2} \tilde{c} N_{L}\right) \frac{v_{\infty}}{L},
$$

(with the obvious definition for $G\left(\alpha N_{L}\right)$ ) where $\ell_{\mathrm{f}}$ is the relavant friction lengthscale. Note that to obtain this equation one needs to use the evolution equation for the long-string correlation length $L$. 
We should also say at this stage that once the network leaves the frictiondominated regime and strings become relativistic other mechanisms (notably radiation) can cause charge losses. Thus we do not expect our toy model to provide quantitatively correct answers in this regime, but we do expect it to provide reliable order-of-magnitude estimates.

\section{EQUILIBRATION AND SCALING}

We start by discussing the case of neutral currents, in which $\ell_{\mathrm{f}}$ is given by (2.3). Analysis of (2.8) together with the evolution equations for the long-string network reveals two types of behaviour. Firstly, if $f$ is small (that is, equilibration is ineffective) then $N_{L}$ grows without limit. In the particular case $f=0, N_{L} \propto t$ once the long-string network has reached the linear scaling regime, meaning that $\xi \propto$ const. On the other hand, for

$$
f>f_{\min }=3-\frac{2 \tilde{c}}{k+\tilde{c}} \sim 1.88
$$

(where we have used the scaling values of $\tilde{c}$ and $k$ obtained from numerical simulations in the radiation era - see [5] for a discussion) the late time behaviour is $N_{L} \propto$ const., $\xi \propto t$, that is the superconducting correlation length is scaling linearly just like the long-string correlation length; the scaling value of $N_{L}$ has a fairly weak dependence on $f$, with smaller $N_{L}$ 's corresponding to larger $f$ 's as expected.

Note that the fact that correlations cannot obviously be established faster than the speed of light (that is, we must have $\xi \leq t$ ), means that there is a maximum value allowed for $f$, which at late times (in the radiation era) can be written

$$
f<f_{\max }=3+\frac{4(1-2 \tilde{c})}{k^{1 / 2}(k+\tilde{c})^{3 / 2}} \sim 22.4 .
$$

\section{TWO EXAMPLES}

In figure 2 we plot the result of the numerical integration of (2.8) for GUT string networks in the friction-dominated epoch, for initial conditions representative of string-forming and superconducting phase transitions of first and second order, for the cases $f=0$ and $f=3$, which are representative of the cases where equilibration is ineffective and effective, respectively. We are assuming that both of these phase transitions occur at around the same (GUT) energy scale. It was also assumed that the value of $\alpha$ in the linear scaling regime is $\alpha_{s c} \sim 10^{-3}$ (see Martins and Shellard (5).

The differences between the two cases are considerable. If there is no equilibration mechanism $(f=0), \xi$ is conformally stretched during the stretching regime (just like the long-string correlation length, $L$ ), and so $N_{L}$ is approximately constant. However, as inter-commutings start creating new regions $N_{L}$ begins to increase, growing as $t^{3 / 2}$ during the Kibble regime and eventually (once the network reaches the linear scaling regime) ends up growing as $N_{L} \propto t$, which corresponds to $\xi \propto$ const. As expected, in this case the network keeps a 'memory' of its initial conditions. On the other hand, if there is an equilibration mechanism $(f=3)$ then $N_{L}$ decreases while the network is being conformally stretched. In the Kibble regime, the increased number of inter-commutings again drives $N_{L}$ up, and after $\alpha$ has evolved into its linear regime value $\xi$ itself reaches a scaling value and hence $N_{L}$ becomes a constant. 


\section{PLASMA EFFECTS}

If the cosmic strings interact with a plasma there will be a further damping term, with the corresponding friction lengthscale being given by

$$
\ell_{\mathrm{j}}=\frac{\mu}{\rho^{1 / 2} J},
$$

where $\rho$ is the plasma density and $J$ is the string current; at a scale $r$ this is given by

$$
J(r)=e \frac{N_{r}^{1 / 2}}{r},
$$

$N_{r}$ being the number of uncorrelated current regions at that scale.

We must therefore determine which of the two damping lengthscales is dominant (that is, smaller). It is straightforward to find that at a time $t=x t_{c}\left(t_{c}\right.$ being the epoch of string formation)

$$
\frac{\ell_{\mathrm{j}}}{\ell_{\mathrm{f}}}=\left(\frac{32 \pi}{3}\right)^{1 / 2} \frac{\gamma x^{1 / 2}}{d e N_{L}^{1 / 2}(G \mu)^{1 / 2}},
$$

where $L=\gamma t$ and $d=4 \pi(\pi \mathcal{N} / 45)^{1 / 2},(\mathcal{N}$ being the number of effectively massless degrees of freedom). We therefore require this ratio to be less than unity for plasma effects to be dominant.

Now, what initial currents do we expect at $t_{c}$ ? If plasma effects are to be important, $\ell_{\mathrm{j}}<\xi<t$ at $t_{c}$; note that $\ell_{\mathrm{j}}$ depends on $\xi$, but we can still solve consistently for the minimum allowed $\ell_{\mathrm{j}}$, which will produce the largest current. Using these bounds we find in the GUT case

$$
4 \leq\left(\frac{\ell_{\mathrm{j}}}{\ell_{\mathrm{f}}}\right)_{t c} \leq 60,
$$

so that plasma effects are initially subdominat; the lower bound is $G \mu$-independent, whereas the upper one varies as $(G \mu)^{-1 / 2}$. However, note that the lower bound depends on $\mathcal{N}$, with a ratio of four corresponding to a minimal GUT model $\mathcal{N} \sim$ 106.75; one would need $\mathcal{N} \sim 10^{4}$ for plasma effects to become dominant-but even so, this would only happen in the 'extreme' case of a strongly first order stringforming phase transition and a second order superconducting phase transition.

Thus we can in general study the initial stages of superconducting string evolution with (2.8) and the Aharonov-Bohm friction lenghtscale. But then the evolution of the currents is such that the ratio (5.3) is at most approximately constant - this happens in the 'extreme' case of no equilibration, $f=0$, in which $N_{L}$ respectively behaves as $N_{L} \propto$ const., $N_{L} \propto t^{3 / 2}$ and $N_{L} \propto t$ in the stretching, Kibble and linear scaling regimes. If there is equilibration (that is $f \neq 0$ ) then $N_{L}$ grows more slowly than the above and the plasma damping effect loses importance relative to Aharonov-Bohm scattering (that is, $\ell_{\mathrm{j}}$ grows faster than $\ell_{\mathrm{f}}$ ).

Hence we find that plasma effects are in general subdominant. However, a way of avoiding these constraints is to have further current-building mechanisms, such as background magnetic field - this is in fact commonly assumed (and not very well explained) in most of the existing litterature [13]. What we have shown is that if no such charging mechanism exists than the currents on the strings are much smaller. 


\section{CONCLUSIONS}

In this paper we have constructed a simple 'toy model' for the evolution of currents on cosmic strings, based on the dynamics of a 'superconducting correlation length. The different dynamical processes affecting it were considered, and a simple study of the solutions of the model has revealed that the process of equilibration is crucial in the evolution of the currents. We have found that a fairly efficient equilibration mechanism is needed for the superconducting correlation length to scale. Whether or not such a mechanism is available in realistic model is an issue which cannot be addressed at the moment - a detailed numerical investigation will clearly be needed for that.

We have also studied the importance of plasma damping effects on the strings. We find that these are typically negligible when compared to the 'usual' AharonovBohm scattering. However, an exception to this might happen in the presence of background magnetic fields, either of 'primordial' origin or generated (by a dynamo mechanism, say) after protogalaxies start to collapse (an example of the later is the Chudnivsky-Vilenkin scenario [13]). Even in this case, for typical values of cosmological or astrophysical magnetic fields, the two damping lengthscales are not too different.

While this paper was being prepared, a rather less accurate discussion of plasma effects on string dynamics appeared in the litterature [14. This is in broad agreement with our results if one allows for the fact that in 14 no consideration is given to the dynamics of currents - in fact current is simply treated as a constant.

Our extended quantitative model allows reliable estimates of the currents on strings to be made at all times. Thus a more detailed analysis of some of the cosmological scenarios involving superconducting cosmic strings becomes possible. An outstanding example the question of the abundance of vorton remnants - we will address it in a forthoming publication [9].

[1] E. Witten, Nucl. Phys. B249, 557 (1985).

[2] A. Vilenkin \& E. P. S. Shellard, 'Cosmic Strings and other Topological Defects', Cambridge University Press (1994).

[3] R. L. Davis \& E. P. S. Shellard, Nucl. Phys. B249, 557 (1989).

[4] C. J. A. P. Martins \& E. P. S. Shellard, Phys. Rev. D53, 575 (1996).

[5] C. J. A. P. Martins \& E. P. S. Shellard, Phys. Rev. D54, 2535 (1996).

[6] C. J. A. P. Martins \& E. P. S. Shellard, 'Averaged methods for vortex-string evolution', cond-mat/9607093, submitted to Phys. Rev. B.

[7] C. J. A. P. Martins, Phys. Rev. D55, 5208 (1997).

[8] P. P. Avelino, R. R. Caldwell \& C. J. A. P. Martins, 'Cosmic Strings in an Open Universe: Quantitative Evolution and Observational Consequences', submitted to Phys. Rev. D.

[9] C. J. A. P. Martins \& E. P. S. Shellard, 'Conditions for Vorton Formation', in preparation.

[10] R. Rohm, Ph.D. thesis, Princeton University (1985);

P. de Sousa Gerbert \& R. Jackiw, Comm. Math. Phys. 124, 229 (1988);

M. G. Alford \& F. Wilczek, Phys. Rev. Lett. 62, 1071 (1989).

[11] P. Laguna \& R. A. Matzner, Phys. Rev. D41, 1751 (1990).

[12] D. Austin, E. J. Copeland and T. W. B. Kibble, Phys. Rev. D48, 5594 (1993).

[13] E. Chudnovsky \& A. Vilenkin, Phys. Rev. Lett. 61, 1043 (1988). 
[14] K. Dimopoulos \& A. C. Davis, hep-ph/9705302.
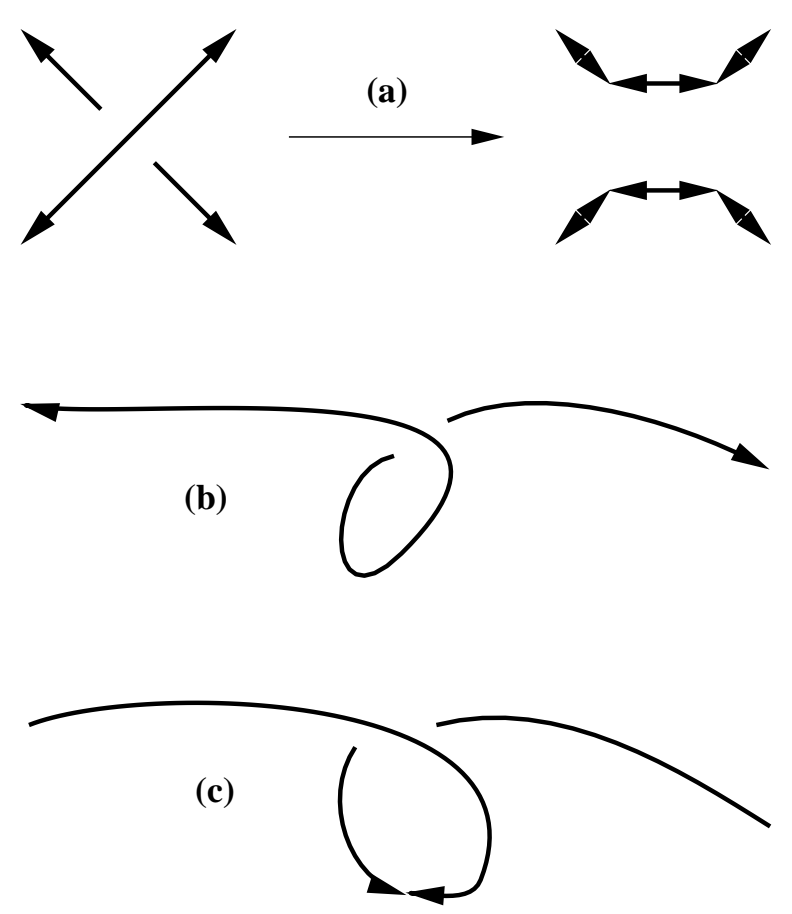

FIG. 1. Some relevant inter-commuting configurations. The arrows mark the limits of regions with correlated currents. Plot (a) shows a typical inter-commuting creating four new current regions, while (b-c) show than on scales smaller than the current correlation length loop production may (c) or may not (b) remove current regions from the long-string network. 

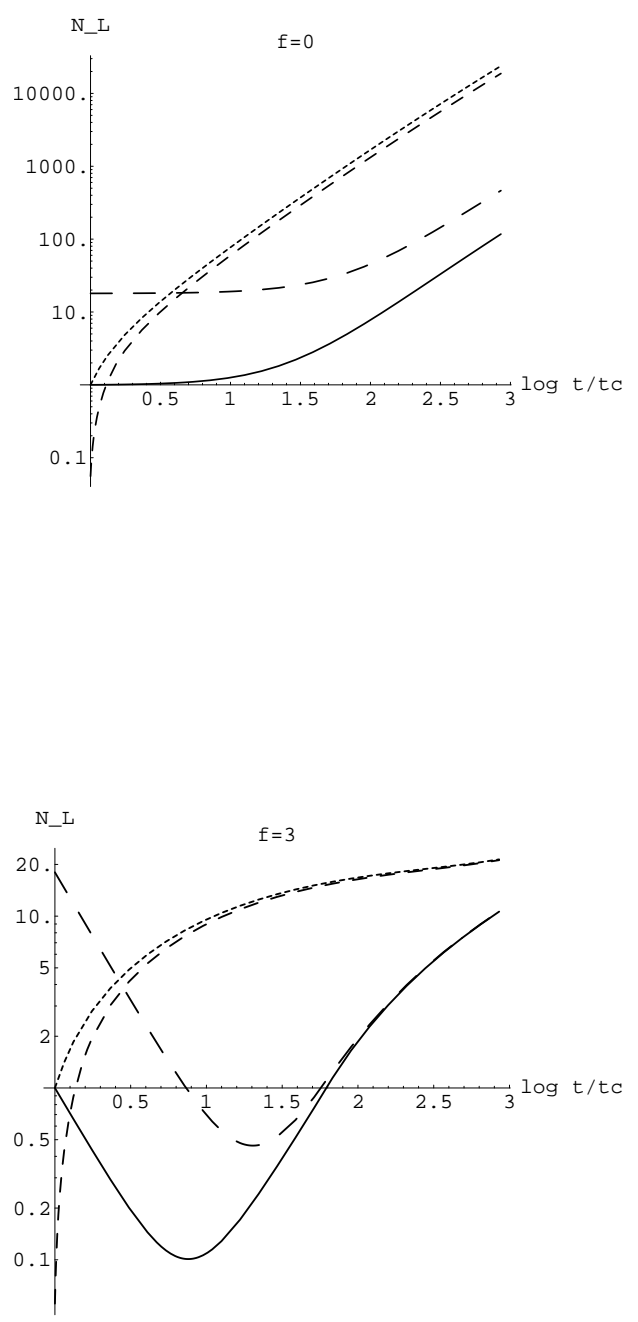

FIG. 2. The evolution of the number of uncorrelated current regions per long-string correlation length, $N_{L}$, for the cases $f=0$ (top) and $f=3$ (bottom) assuming that the orders of the string-forming and superconducting phase transitions are respectively: 1st \& 1st (solid lines), 1st \& 2nd (long dashed), 2nd \& 1st (short dashed) and 2nd \& 2nd (dotted). Time is in orders of magnitude from the epoch of string formation. 